\title{
Virtual Knot Groups and Combinatorial Knots
}

\section{José Gregorio Rodríguez}

Universidad Nacional de Colombia, Sede Medellín.

E-mail address: jgrodrig@unalmed.edu.co

\section{Margarita Toro}

Universidad Nacional de Colombia, Sede Medellín.

E-mail address: mmtoro@unalmed.edu.co

\begin{abstract}
Kauffman [16] and Kim [17] defined the group of a virtual knot by extending, in a natural way, the Wirtinger presentation of the fundamental group of classical knot. In this paper we present the group of a virtual knot by using the concept of combinatorial knot, introduced by Toro [21]. We show the advantages of this approach, that provides natural algorithms. We present examples of combinatorial knots whose groups have properties that are false, or unknown, in the category of the classical knots.

Keywords: Knots, Virtual Knots, Combinatorial Knots, Knot Groups, Virtual Knot Groups.
\end{abstract}

\section{Introduction}

In this paper we study the group of a virtual knot by using the concept of combinatorial knot. The group of a virtual knot was introduced by Kauffman [16] as a generalization of the group of a classical knot. This definition relies on a virtual knot diagram, so we do not have easy algorithms and computations are cumbersome. One of the advantages of using combinatorial knots to approach virtual knots is that we are not required to use diagrams and the definitions and proofs are presented in algorithmic form, easy to implement using a symbolic computation software such as Mathematica. We used combinatorial knots, instead of Gauss codes, introduced by Kauffman [16], because the combinatorial knot theory gives us an easy way to extend definitions and invariants from the classical knot theory to virtual knot theory. The second author has used combinatorial knots

\footnotetext{
${ }^{0}$ Partially supported by Colciencia, code 436-2007 and DIME.
} 
to obtain an algorithm to construct an $n$-butterfly of a classical knot, see [11] and [21]. In our research, we use combinatorial knots, not only as a computational tool, but also as theoretical one, for instance, using combinatorial knots we found an easy way to compute an invariant that provides a necessary condition for a virtual knot to be a classical knot, see [22].

The paper is organized as follows. In section 2 we present a brief review of virtual knots [16] and combinatorial knots [21] and we sketch the proof that the virtual knot category is equivalent to the combinatorial knot category. Using this fact, in the rest of the paper we use combinatorial knots to present all the definitions and results and we do not differentiate between virtual knots and combinatorial knots. Section 3 provides the definition of the group of a combinatorial knot by using a presentation that extends the Wirtinger presentation of a classical knot. Given the correspondence between combinatorial knots and virtual knots, we define the group of a virtual knot. This definition is the same as the one given by Kauffman. In Section 4 we present properties of combinatorial knot groups and give several relevant examples. We present Kim's result [17], in which he gave conditions to determine when $G$ is the fundamental group of a virtual knot. In the case that $G$ corresponds to the group of a virtual knot, we give an algorithm to construct a combinatorial knot from the group presentation. Our proof of this result and the algorithm we get, show the advantages and power of working with combinatorial knots.

In the paper we work only with combinatorial knots and virtual knots, but there exists the concept of combinatorial link that corresponds to virtual link, and all our work can be extended to the group of a virtual link. It is important to note that there are properties that are different in the case of virtual link groups and this topic is the subject of our current research.

\section{Virtual knots and combinatorial knots}

The concept of a virtual knot was introduced by Kauffman [16] as a generalization of classical knot diagram. A virtual knot diagram is an oriented 4-valent planar connected graph, whose crossings are classified according to Figure 1a. An example of a virtual knot diagram is shown in Figure 1b.

The moves of virtual knot diagrams, illustrated in Figure 2 are called generalized Reidemeister moves. Two virtual knot diagrams $D$ and $D^{\prime}$ are equivalent if $D$ can be transformed into $D^{\prime}$ by a finite number of generalized Reidemeister moves. A virtual knot is an equivalence class of virtual knot diagrams.

Goussarov, Polyak and Viro [8] showed that the entire theory of classical knots is contained in the theory of virtual knots. Kauffman [16] gave examples of non classical virtual knots. In this sense virtual knot theory is 


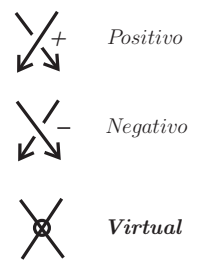

$a$

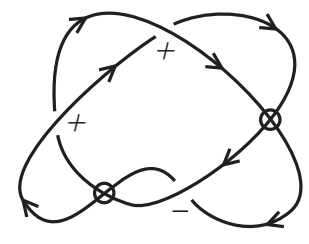

$b$

Figure 1. Cossing types.
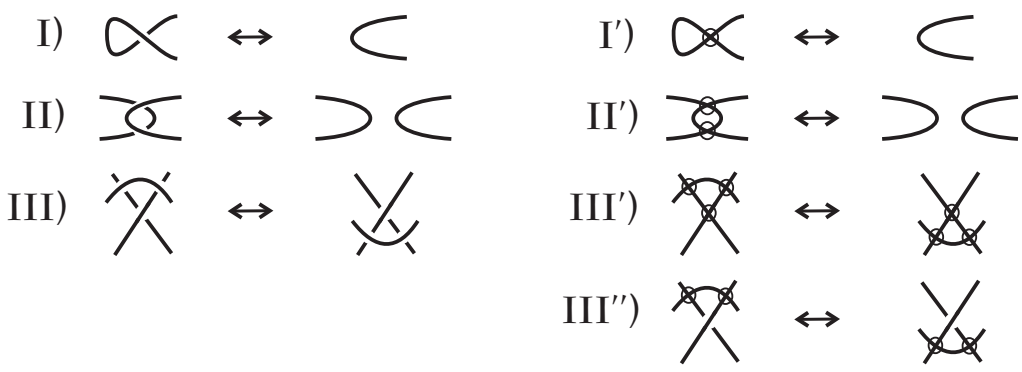

FiguRE 2. I), II) and III) are the classical Reidemeister moves.

a nontrivial extension of the classical theory. Many invariants of classical knots have been extended to invariants of virtual knots, among them, the group of a classical knot.

Combinatorial knot theory was proposed by Toro [21] as a computational extension of knot theory. Figure 3 shows a diagram of a classical knot and its combinatorial knot.

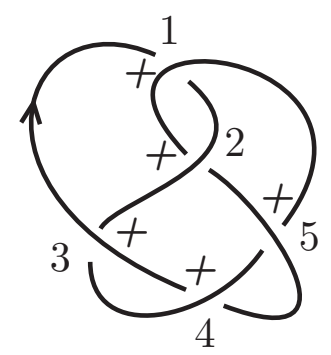

Figure 3. ((-1, 2, -3, 4, -5, 1, - 2, 5, -4,3), (1, 1, 1, 1, 1)) 
Definition 1. A knot code of $n$ crossings is defined as

$$
\left(\left(i_{1}, i_{2}, \cdots, i_{2 n}\right),\left(e_{1}, \cdots, e_{m}\right)\right)
$$

where, as sets, $\left\{i_{1}, i_{2}, \cdots, i_{2 n}\right\}=\left\{a_{1}, \cdots, a_{n}\right\} \cup\left\{-a_{1}, \cdots,-a_{n}\right\}$,

$a_{1}, \ldots, a_{n} \in \mathbb{N}, m \geq \max \left\{a_{1}, a_{2}, \cdots, a_{n}\right\}$ and $e_{j} \in\{1,-1\}, j=1,2, \cdots, m$.

The sequences $\left(i_{1}, i_{2}, \cdots, i_{2 n}\right)$ and $\left(e_{1}, \cdots, e_{m}\right)$ are called crossing list and sign list, respectively.

Two knot codes $K_{1}$ and $K_{2}$ are said to be equivalent if $K_{1}$ can be transformed into $K_{2}$ by a finite number of the following moves:

TYPE A: (Rotation)

$\left(\left(i_{1}, i_{2}, \cdots, i_{2 n}\right),\left(e_{1}, \cdots, e_{m}\right)\right) \approx\left(\left(i_{2}, i_{3}, \cdots, i_{2 n}, i_{1}\right),\left(e_{1}, \cdots, e_{m}\right)\right) \approx$

$\approx \cdots \approx\left(\left(i_{2 n}, i_{1}, i_{2}, \cdots, i_{2 n-1}\right),\left(e_{1}, \cdots, e_{m}\right)\right)$.

TYPE B: (Renumbering)

$\left(\left(i_{1}, i_{2}, \cdots, i_{2 n}\right),\left(e_{1}, \cdots, e_{m}\right)\right) \approx\left(\left(j_{1}, j_{2}, \cdots, j_{2 n}\right),\left(\varepsilon_{1}, \cdots, \varepsilon_{l}\right)\right)$

if there exist a bijective map $\varphi:\left\{i_{1}, \cdots, i_{2 n}\right\} \rightarrow\left\{j_{1}, \cdots, j_{2 n}\right\}$, such that, for every $k=1,2, \cdots, n, \varphi\left(-i_{k}\right)=-\varphi\left(i_{k}\right)$ and $\varepsilon_{\left|\varphi\left(i_{k}\right)\right|}=e_{\left|i_{k}\right|}$.

TYPE C: (Simplification)

$$
\left(\left(i_{1}, i_{2}, \cdots, i_{2 n}\right),\left(e_{1}, \cdots, e_{m}, e_{m+1}\right)\right) \approx\left(\left(i_{1}, i_{2}, \cdots, i_{2 n}\right),\left(e_{1}, \cdots, e_{m}\right)\right),
$$

if $m \geq\left|i_{j}\right|$ for all $j=1, \cdots, 2 n$.

TYPE I:

$\left(\left(i_{1}, \cdots, i_{r-1}, i_{r},-i_{r}, i_{r+2}, \cdots, i_{2 n}\right),\left(e_{1}, \cdots, e_{m}\right)\right) \approx$

$\left(\left(i_{1}, \cdots, i_{r-1}, i_{r+2}, \cdots i_{2 n}\right),\left(e_{1}, \cdots, e_{m}\right)\right)$.

TYPE II:

$\left(\left(i_{1}, i_{2}, \cdots, i_{k-1}, h, j, i_{k+2}, \cdots, i_{t-1},-h,-j, i_{t+2}, \cdots, i_{2 n}\right),\left(e_{1}, \cdots, e_{m}\right)\right)$

$\approx\left(\left(i_{1}, i_{2}, \cdots, i_{k-1}, i_{k+2}, \cdots, i_{t-1}, i_{t+2}, \cdots i_{2 n}\right),\left(e_{1}, \cdots, e_{m}\right)\right)$

or

$\left(\left(i_{1}, i_{2}, \cdots, i_{k-1}, h, j, i_{k+2}, \cdots, i_{t-1},-j,-h, i_{t+2}, \cdots, i_{2 n}\right),\left(e_{1}, \cdots, e_{m}\right)\right)$

$\approx\left(\left(i_{1}, i_{2}, \cdots, i_{k-1}, i_{k+2}, \cdots, i_{t-1}, i_{t+2}, \cdots i_{2 n}\right),\left(e_{1}, \cdots, e_{m}\right)\right)$

if and only if $j . h>0$ and $e_{|j|}=-e_{|h|}$.

TYPE III:

$\left(\left(i_{1}, \cdots, j, h, \cdots, p, q, \cdots, v, w, \cdots, i_{2 n}\right),\left(e_{1}, \cdots, e_{m}\right)\right) \approx$

$\approx\left(\left(i_{1}, \cdots, h, j, \cdots, q, p, \cdots, w, v, \cdots, i_{2 n}\right),\left(e_{1}, \cdots, e_{m}\right)\right)$ if and only if $j . h>0, p \neq q, w \neq v$ and $\{p, q, v, w\}=\{-j,-h, k,-k\}$, for some crossing $k$.

The relations Type I, II and III are denominated Reidemeister moves, in a similar way to the classical case.

Note that in the type I and II moves we do not change the list of signs. In this way, we do not need to renumber the crosses and it could be information 
about crossings that do not exist anymore. This is a special feature of combinatorial knots, that is extremely useful in computations. Note also that we have only a set of Reidemeister moves.

A combinatorial knot is defined as an equivalence class of knot codes under the relation $\approx$. A combinatorial knot is called trivial or unknot if it is equivalent to $((),())$.

The category of combinatorial knots is equivalent to the category of virtual knots (and also to the category of Gauss diagrams and abstract knots), see $[22],[15],[8]$ and [16].

We describe briefly a biyection between the set of virtual knot diagrams and the set of combinatorial knots. This correspondence extends to a biyective correspondence between the category of virtual knots and the category of combinatorial knots, see [22] for details.

Let $K$ be a virtual knot diagram. We label its classical crossings with the numbers $1,2, \ldots, n$, where $n$ is the number of classical crossing of $K$. We take a point over $K$, not a crossing, and we follow the diagram writing down the list of crossing labels, with the convention that if we pass through an undercrossing $i$, we add $-i$ to the list, but if we pass through an overcrossing $i$, we add $i$ to the list. The resulting collection is called crossing list of $K$. We construct also the list $\left(e_{1}, e_{2}, \ldots, e_{n}\right)$, where $e_{i}=1$ if the crossing $i$ is positive or $e_{i}=-1$ if it is negative. This list is called list of signs of $K$. The list formed by these two lists is a knot code that represents the virtual knot diagram $K$. It is denoted $\sigma_{K}$.

Now, we present a sketch of a construction of a virtual knot diagram from a knot code. Let $\sigma=\left(\left(i_{1}, i_{2}, \cdots, i_{2 n}\right),\left(e_{1}, \cdots, e_{m}\right)\right)$ be a knot code, where $\left\{i_{1}, i_{2}, \cdots, i_{2 n}\right\}=\left\{a_{1}, \cdots, a_{n}\right\} \cup\left\{-a_{1}, \cdots,-a_{n}\right\}$. Let $I_{\sigma}$ be a graph with vertices given by the set $\left\{a_{1}, \cdots, a_{n}\right\}$ and its edges given by the set $\left\{\left(i_{1}, i_{2}\right), \ldots,\left(i_{n}, i_{1}\right)\right\}$, where $\left(i_{k}, i_{k+1}\right)$ connects the vertices $a_{i}$ and $a_{j}$ if and only if $\left|i_{k}\right|=a_{i}$ and $\left|i_{k+1}\right|=a_{j}$. We classify the vertices of $I_{\sigma}$ according to Figure $4 a$.

Now, we consider the transformations shown in Figure $4 b$. If we take a plane realization of $I_{\sigma}$ and apply these transformations to each vertex, we obtain a virtual knot diagram corresponding to the combinatorial knot $\sigma$. It is denoted $K_{\sigma}$.

As an example, for the combinatorial knot $\sigma=((-1,-2,3,1,-3,2),(-1$, $1,1)$ ), we get the diagram $K_{\sigma}$ shown in Figure 5 .

Definition 2. A combinatorial knot $\sigma$ is called admissible or classical if $K_{\sigma}$ is equivalent to a diagram without virtual crossings.

The combinatorial knot $((1,-2,7,-4,5,-1,2,-5,4,-7)),(-1,-1,1,-1$, $1,1,-1)$ ) is classical, because it represents the classical knot of Figure 6 . 


$$
\begin{gathered}
\underset{\left.\left(a,-a_{i}\right)\right|_{\left(c, a_{i}\right)} ^{\uparrow\left(-a_{i}, b\right)}\left(a_{i}, d\right)}{\longrightarrow} a_{i} \\
e_{a_{i}}=1
\end{gathered}
$$

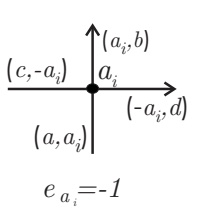

$a$

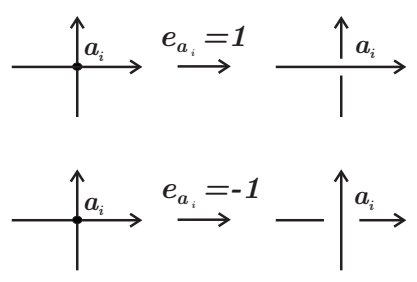

$b$

FiguRE 4. Transforming vertices to classical crossing.
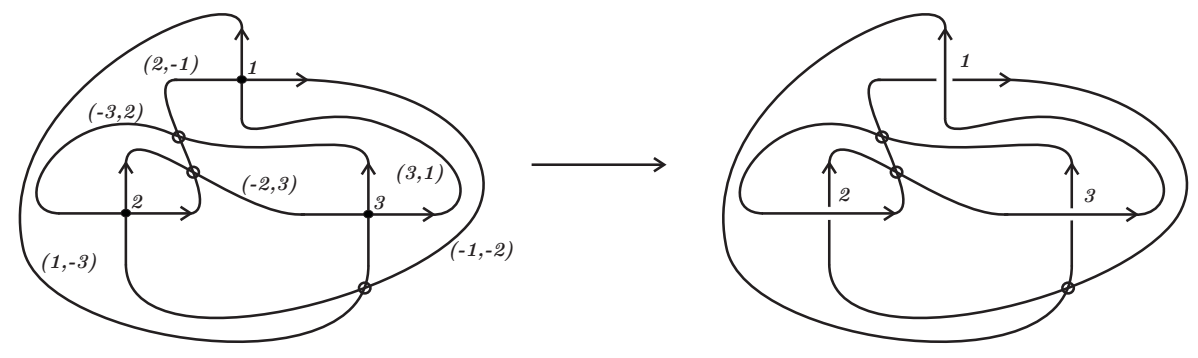

Figure 5. Diagram $K_{\sigma}$ for $\sigma=((-1,-2,3,1,-3,2),(-1,1,1))$.

This combinatorial knot is equivalent to the combinatorial knot $((-1,2,-3,1,-4,5,-2,3,-5,4),(-1,-1,-1,-1,-1))$.

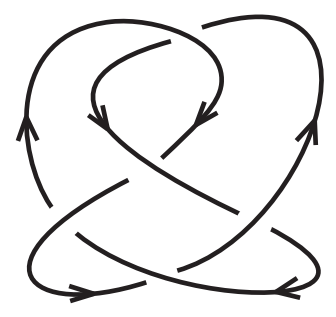

Figure 6. Clasical knot.

We will show in Example 1 that the combinatorial knot in Figure 5 is not classical, using the group of a combinatorial knot. 


\section{Definition of Combinatorial Knot Group}

In this section we introduce the concept of combinatorial knot group and by using the correspondence between virtual knots and combinatorial knots, we recover Kaufmann's definition of virtual knot group.

Let $\sigma=\left(\left(i_{1}, i_{2}, \cdots, i_{2 n}\right),\left(e_{1}, e_{2}, \cdots, e_{m}\right)\right)$ be a combinatorial knot and let $a_{1}, \ldots, a_{n} \in \mathbb{Z}^{+}$be such that $\left\{i_{1}, i_{2}, \cdots, i_{2 n}\right\}=\left\{-a_{1},-a_{2}, \ldots,-a_{n}\right\} \cup$ $\left\{a_{1}, a_{2}, \ldots, a_{n}\right\}$. Without lost of generality we suppose that

$$
\sigma=\left(\left(-a_{1}, i_{2}, \cdots i_{r},-a_{2}, i_{r+2}, \ldots,-a_{n}, i_{t+2}, \ldots, i_{2 n}\right),\left(e_{1}, e_{2}, \cdots, e_{m}\right)\right),
$$

and that between $-a_{i}$ and $-a_{i+1}, i=1, \ldots, n-1$, there are no numbers or only positive numbers. We define the arcs of $\sigma$ as the subsequences $S_{a_{i}}=$ $\left(-a_{i}, \cdots,-a_{i+1}\right), i=1,2, \ldots, n-1$ and $S_{a_{n}}=\left(-a_{n}, i_{t+2} \cdots, i_{2 n},-a_{1}\right)$.

For example, for the combinatorial knot $\sigma=((-1,2,-3,-4,1,-2,4,3)$, $(1,-1,1,1))$ the arcs are $S_{1}=(-1,2,-3), S_{3}=(-3,-4), S_{4}=(-4,1,-2)$ and $S_{2}=(-2,4,3,-1)$.

Definition 3. Let $\sigma=\left(\left(i_{1}, i_{2}, \cdots, i_{2 n}\right),\left(e_{1}, e_{2}, \cdots, e_{m}\right)\right)$ be a non trivial knot code as in (3.1) and let $S_{a_{1}}, \ldots, S_{a_{n}}$ to be its arcs. We define the group of $\sigma$ as

$$
G(\sigma)=\left\langle S_{a_{1}}, S_{a_{2}}, \cdots, S_{a_{n}}: r_{1}, r_{2}, \cdots, r_{n}\right\rangle,
$$

where $r_{1}=S_{t_{1}}^{-e_{a_{1}}} S_{a_{n}} S_{t_{1}}^{e_{a_{1}}} S_{a_{1}}^{-1}, \quad r_{j}=S_{t_{j}}^{-e_{a_{j}}} S_{a_{j-1}} S_{t_{j}}^{e_{a_{j}}} S_{a_{j}}^{-1}$, for $j=2, \ldots, n$, and $a_{j} \in S_{t_{j}}, t_{j} \in\left\{a_{1}, \ldots, a_{n}\right\}, j=1,2, \ldots, n$.

We define the group of $((),())$ as $\mathbb{Z}$.

It is straightforward to prove that the group is invariant under moves A, $\mathrm{B}, \mathrm{C}$ and Reidemeister moves. This allows us to define the group of a combinatorial knot.

To simplify notation, in the rest of the paper we will take all combinatorial knots to be of the form

$$
\left(\left(i_{1}, i_{2}, \cdots, i_{2 n}\right),\left(e_{1}, \cdots, e_{n}\right)\right),
$$

with $\left\{i_{1}, i_{2}, \cdots, i_{2 n}\right\}=\{1, \cdots, n\} \cup\{-1, \cdots,-n\}$.

Definition 4. The group of a combinatorial knot $K$ is defined by $G(K)=$ $G(\sigma)$, where $\sigma$ is any knot code in the class of $K$. For a virtual knot $K$ we define the group of $K$ as $G(K)=G\left(\sigma_{K}\right)$, where $\sigma_{K}$ is a knot code that represents $K$.

This definition of the group of a virtual knots corresponds to the definition given by Kauffman and Kim, see [16] and [17]. In the case of a classical 
knot $K$, it corresponds to the fundamental group of the knot complement, $\pi\left(S^{3}-K\right)$.

\section{Properties of Combinatorial Knot Groups}

In this section we establish some properties of the group of a combinatorial knot and we give several examples.

Lemma 1. Let $K$ be a combinatorial knot. If $G=G(K)$ then $G_{a b} \cong \mathbb{Z}$.

Proof. We know that if $K$ is a combinatorial knot then $G(K)$ has presentation

$$
G(K)=\left\langle S_{1}, S_{2}, \cdots, S_{n}: r_{1}, r_{2}, \cdots, r_{n}\right\rangle,
$$

where $r_{j}=S_{t_{j}}^{-e_{j}} S_{j-1} S_{t_{j}}^{e_{j}} S_{j}^{-1}, j=2, \ldots, n$ and $r_{1}=S_{t_{1}}^{-e_{1}} S_{n} S_{t_{1}}^{e_{1}} S_{1}^{-1}$. A presentation for $G_{a b}$ is

$$
\left\langle S_{1}, S_{2}, \cdots, S_{n}: r_{1}, r_{2}, \cdots, r_{n},\left[S_{i}, S_{j}\right], i, j=1,2, \ldots, n\right\rangle,
$$

where $\left[S_{i}, S_{j}\right]=S_{i} S_{j} S_{i}^{-1} S_{j}^{-1}$. Therefore,

$$
\begin{aligned}
G_{a b} & \cong\left\langle S_{1}, S_{2}, \cdots, S_{n}: r_{1}, r_{2}, \cdots, r_{n}, S_{i} S_{j}=S_{j} S_{i}, i, j=1,2, \ldots, n\right\rangle \\
& \cong\left\langle S_{1}, S_{2}, \cdots, S_{n}: S_{1}=S_{2}, S_{2}=S_{3}, \ldots, S_{n-1}=S_{n}\right\rangle \\
& \cong\left\langle S_{i}\right\rangle, i=1,2, \ldots, n .
\end{aligned}
$$

For a classical combinatorial knot $K$, the group $G(K)$ has a known and important topological interpretation.

Theorem 1. If $K$ is a classical combinatorial knot and $K^{*}$ is a knot in $S^{3}$ that is represented by $K$ then $G(K)$ is the fundamental group $\pi_{1}\left(S^{3}-K^{*}\right)$.

In the rest of the paper we do not distinguish between a classical combinatorial knot and the corresponding knot in $S^{3}$.

The following result is central in classical knot theory, but it is not true in combinatorial knot theory, as Example 1 shows.

Theorem 2. [6] If $K$ is a classical combinatorial knot such that $G(K)$ is isomorphic to $\mathbb{Z}$, then $K$ is trivial.

Example 1. For the combinatorial knot $L=((-1,-2,3,1,-3,2),(-1,1$, 1)) we have

$$
G(L)=\left\langle S_{1}, S_{2}, S_{3}: S_{2} S_{3} S_{2}^{-1} S_{1}^{-1}, S_{3}^{-1} S_{1} S_{3} S_{2}^{-1}, S_{2}^{-1} S_{2} S_{2} S_{3}^{-1}\right\rangle \cong \mathbb{Z} .
$$

However, it is known that $L$ is not equivalent to the trivial knot, see [10] and [23]. For the proof they use the polynomial invariant $P$, see also [22]. So, $L$ is not a classical combinatorial knot. 
Example 2. Consider $K=((-1,2,3,-4,-3,4,1,-2),(1,1,-1,-1))$. The arcs of $K$ are: $S_{1}=(-1,2,3,-4), S_{2}=(-2,-1), S_{3}=(-3,4,1,-2)$ and $S_{4}=(-4,-3)$. The relators for $G(K)$ are:

therefore,

$$
\begin{array}{ll}
r_{1}: S_{3}^{-1} S_{2} S_{3} S_{1}^{-1}, & r_{2}: S_{1}^{-1} S_{3} S_{1} S_{2}^{-1}, \\
r_{3}: S_{1} S_{4} S_{1}^{-1} S_{3}^{-1}, & r_{4}: S_{3} S_{1} S_{3}^{-1} S_{4}^{-1},
\end{array}
$$

$$
G(K)=\left\langle S_{2}, S_{3}: S_{2} S_{3} S_{2}=S_{3} S_{2} S_{3}\right\rangle \cong\left\langle y, z: z^{3}=y^{2}\right\rangle .
$$

As $G(K)$ is not isomorphic to $\mathbb{Z}$, see [1], we obtain that $K$ is not the trivial $k n o t$. We will probe in the next section that $K$ is not a classical knot.

\subsection{The Over Presentation.}

Definition 5. Let $\sigma=\left(\left(i_{1}, i_{2}, \cdots, i_{2 n}\right)\right),\left(e_{1}, e_{2}, \cdots, e_{n}\right)$ be a non trivial knot code and let $S_{1}, S_{2}, \ldots, S_{n}$ be the arcs of $\sigma$. We denote by $\left|S_{i}\right|$ the cardinality of $S_{i}$. We say that $S_{i}$ is a bridge of $\sigma$ if $\left|S_{i}\right|>2$. For a knot code $\sigma$ the number of bridges is called the bridge number, and is denoted by $\operatorname{br}(\sigma)$. For a combinatorial knot $K$ its bridge number is defined by $\operatorname{br}(K)=\min \{b r(\sigma):[\sigma]=K\}$.

\section{Remark 1.}

(b) If $K$ is a classical combinatorial knot and $\operatorname{br}(K)=1$, then $K$ is trivial.

(c) In general, if $b r(K)=1$, not necessary $K$ is trivial. For example, for $K=((1,-2,-1,2),(1,1))$ we have that br $(K)=1$ but it is possible to prove that $K$ is not trivial, see [22].

Let $K$ a combinatorial knot and $y_{t}=(-t, \ldots,-(t+1)), y_{t+1}=(-(t+p+$ $1), \ldots,-(t+p+2))$ be the bridges of $K$. Without loss of generality we may assume that $K$ is given by

$$
\begin{aligned}
& K=\left(\left(i_{1}, \cdots,-t, \cdots,-(t+1), \cdots,-(t+p),-(t+p+1), \cdots,-(t+p+\right.\right. \\
& \left.\left.2), \cdots, i_{2 n}\right),\left(e_{1}, \cdots, e_{n}\right)\right)
\end{aligned}
$$

where there is no crossing between $-(t+i)$ and $-(t+i+1)$, for $i=1,2, \ldots, p$. We construct the relator

$$
r_{t}=w_{t}^{-1} y_{t} w_{t} y_{t+1}^{-1}, \quad w_{t}=y_{a_{1}}^{e_{t+1}} y_{a_{2}}^{e_{t+2}} \ldots y_{a_{p}}^{e_{t+p}} y_{a_{p+1}}^{e_{t+p+1}},
$$

where $y_{a_{1}}, y_{a_{2}}, \ldots, y_{a_{p+1}}$ are bridges of $K$ such that $-(t+i) \in y_{a_{i}}, i=$ $1,2, \ldots, p+1$.

The following theorem provides another presentation for $G(K)$ that is called the over presentation. 
Theorem 3. Let $K$ be a non trivial combinatorial knot and let $y_{1}, y_{2}, \ldots$, $y_{m}$ be the bridges of $K$, then

$$
G(K) \cong\left\langle y_{1}, y_{2}, \ldots, y_{m}: r_{1}, \ldots, r_{m}\right\rangle,
$$

where $r_{1}, \ldots, r_{m}$ are as in (4.1).

Proof. Let $K$ be a combinatorial knot, $G(K)=\left\langle S_{1}, S_{2}, \cdots, S_{n}: r_{1}, r_{2}, \cdots\right.$, $\left.r_{n}\right\rangle$ the group of $K$, and $S_{i_{1}}, S_{i_{2}}, \ldots, S_{i_{v}}$ arcs of $K$ such that $\left|S_{i_{j}}\right|=2$, $j=1,2, \ldots, v$. To simplify notation, $x_{j}=S_{i_{j}}, j=1,2, \ldots, v$ and $y_{u}=S_{v+u}$, $u=1, \ldots, m=n-v$ are the bridges of $K$. By Type A movements, we may assume that

$$
\begin{aligned}
& K=\left(\left(i_{1}, \cdots,-t, \cdots,-(t+1), \cdots,-(t+p),-(t+p+1), \cdots,-(t+p+\right.\right. \\
& \left.\left.2), \cdots, i_{2 n}\right),\left(e_{1}, \ldots, e_{m}\right)\right), \\
& y_{t}=(-t, \cdots,-(t+1)), x_{i}=(-(t+i),-(t+i+1)), i=1, \ldots, p \text {, and } \\
& y_{t+1}=(-(t+p+1), \ldots,-(t+p+2)) \text {. Let } y_{a_{1}}, y_{a_{2}}, \ldots, y_{a_{p+1}} \text { be the bridges } \\
& \text { of } K \text { such that }-(t+i) \in y_{a_{i}}, i=1,2, \ldots, p+1 \text {. The relators of } G(\sigma) \text { for } \\
& \text { the crossings }-(t+1),-(t+2), \ldots,-(t+p),-(t+p+1) \text { are: } \\
& \qquad r_{t+1}=y_{a_{1}}^{-e_{t+1}} y_{t} y_{a_{1}}^{e_{t+1}} x_{1}^{-1}, \quad r_{t+i}=y_{a_{i}}^{-e_{t+i}} x_{i-1} y_{a_{i}}^{e_{t+i}} x_{i}^{-1}, i=2, \ldots, p, \\
& r_{t+p+1}=y_{a_{p+1}}^{-e_{t+p+1}} x_{p} y_{a_{p+1}}^{e_{t+p+1}} y_{t+1}^{-1} .
\end{aligned}
$$

From the relator $r_{t+1}$ we get $x_{1}=y_{a_{1}}^{-e_{t+1}} y_{t} S_{a_{1}}^{e_{t+1}}$. Replacing into the relator $r_{t+2}$ we have

$$
x_{2}=y_{a_{2}}^{-e_{t+2}} y_{a_{1}}^{-e_{t+1}} y_{t} y_{a_{1}}^{e_{t+1}} y_{a_{2}}^{e_{t+2}},
$$

from the relator $r_{t+3}$ we get

$$
x_{3}=y_{a_{3}}^{-e_{t+3}} y_{a_{2}}^{-e_{t+2}} y_{a_{1}}^{-e_{t+1}} y_{t} y_{a_{1}}^{e_{t+1}} y_{a_{2}}^{e_{t+2}} y_{a_{3}}^{e_{t+3}}
$$

then

$$
x_{i}=y_{a_{i}}^{-e_{t+i}} \ldots y_{a_{3}}^{-e_{t+3}} y_{a_{2}}^{-e_{t+2}} y_{a_{1}}^{-e_{t+1}} y_{t} y_{a_{1}}^{e_{t+1}} y_{a_{2}}^{e_{t+2}} y_{a_{3}}^{e_{t+3}} \ldots y_{a_{i}}^{e_{t+i}}, i=1,2, \ldots, p .
$$

Now, if we replace $x_{p}$ in the relator $r_{t+p+1}$ we obtain the relator

$$
y_{t+1}=y_{a_{p+1}}^{-e_{t+p+1}} y_{a_{p}}^{-e_{t+p}} \ldots y_{a_{3}}^{-e_{t+3}} y_{a_{2}}^{-e_{t+2}} y_{a_{1}}^{-e_{t+1}} y_{t} y_{a_{1}}^{e_{t+1}} y_{a_{2}}^{e_{t+2}} y_{a_{3}}^{e_{t+3}} \ldots y_{a_{p}}^{e_{t+p}} y_{a_{p+1}}^{e_{t+p+1}} .
$$

If $w_{t}=y_{a_{1}}^{e_{t+1}} y_{a_{2}}^{e_{t+2}} y_{a_{3}}^{e_{t+3}} \ldots y_{a_{p}}^{e_{t+p}} y_{a_{p+1}}^{e_{t+p+1}}$, then $y_{t+1}=w_{t}^{-1} y_{t} w_{t}$. Since $x_{1}, \ldots, x_{p}$ appear only in the relators $r_{t+1}, \ldots, r_{t+p}$ and $r_{t+p+1}$ respectively, then we have

$$
G \cong\left\langle y_{1}, \ldots, y_{m}: \mathbf{r}_{1}, \ldots, \mathbf{r}_{m}\right\rangle, \text { where } \mathbf{r}_{t}=w_{t}^{-1} y_{t} w_{t} y_{t+1}^{-1} \text { for } t=1, \ldots, m .
$$


Example 3. Let $K$ be the combinatorial knot $((1,3,-4,-3,4,2,-1,-2)$, $(1,1,1,1))$. Then

$$
G(K) \cong\left\langle y_{1}, y_{2}: y_{1}^{\left(y_{2} y_{1}\right)} y_{2}^{-1}, y_{2}^{\left(y_{1} y_{2}\right)} y_{1}^{-1}\right\rangle \cong\left\langle y, z: z^{3}=y^{2}\right\rangle ¥ \mathbb{Z} .
$$

Given a combinatorial knot $K$, we define another group, denoted $G_{u}(K)$, as

$$
G_{u}(K)=G\left(K^{*}\right),
$$

where, if $K=\left(\left(i_{1}, i_{2}, \cdots, i_{2 n}\right),\left(e_{1}, e_{2}, \cdots, e_{n}\right)\right.$,

$$
K^{*}=\left(\left(-i_{1},-i_{2}, \cdots,-i_{2 n}\right),\left(-e_{1},-e_{2}, \cdots,-e_{n}\right)\right) .
$$

This group $G_{u}(K)$ is called the under group of $K$ and we sometimes call the group $G(K)$ the over group of $K$.

For combinatorial knots these two groups may not coincide. For the combinatorial knot in Example 2, we have

$$
G_{u}(K) \cong \mathbb{Z} \text { and } G(K)=\left\langle y, z: z^{3}=y^{2}\right\rangle \neq \mathbb{Z} .
$$

In the classical case, however they do coincide.

Lemma 2. If $K$ is a classical knot then $G(K) \cong G_{u}(K)$.

Proof. Let $K$ be a classical knot, then $\pi_{1}\left(S^{3}-K\right) \cong \pi_{1}\left(S^{3}-K^{*}\right)$. Since $G(K)$ and $G\left(K^{*}\right)$ are presentations of the same group, then they are isomorphic.

This lemma allows as to conclude that the combinatorial knot of Example 2 is not a classical combinatorial knot.

4.2. Peripheral system. Let $K=\left(\left(i_{1}, i_{2}, \cdots, i_{2 n}\right),\left(e_{1}, e_{2}, \cdots, e_{n}\right)\right)$ be a combinatorial knot and let $G=G(K)=\left\langle S_{1}, S_{2}, \cdots, S_{n}: r_{1}, r_{2}, \cdots, r_{n}\right\rangle$ be its group.

Let $l=S_{k_{1}}^{e_{1}} S_{k_{2}}^{e_{2}} \cdots S_{k_{n}}^{e_{n}}$, where $i \in S_{k_{i}}, i=1,2, \ldots, n$ and $p=e_{1}+\ldots+e_{n}$. Define $l_{\alpha}=S_{k_{1}}^{e_{1}} S_{k_{2}}^{e_{2}} \cdots S_{k_{n}}^{e_{n}} S_{\alpha}^{-p}$ for all $\alpha=1,2, \ldots, n$.

Lemma 3. With the above notation, $l_{\alpha} \in[G, G]=G^{\prime}$ for all $\alpha=1,2, \ldots, n$.

Proof. Given that $G_{a b} \cong \mathbb{Z}$, by Lemma 1 , we may see $G_{a b}$ as the cyclic group generated by the lateral left class $S_{\alpha} G^{\prime}$ and $S_{i} G^{\prime}=S_{\alpha} G^{\prime}$, for every $i \in\{1,2, \ldots, n\}$, therefore

$$
S_{k_{1}}^{e_{1}} S_{k_{2}}^{e_{2}} \cdots S_{k_{n}}^{e_{n}} G^{\prime}=\left(S_{k_{1}}^{e_{1}} G^{\prime}\right)\left(S_{k_{2}}^{e_{2}}\right) \ldots\left(S_{k_{n}}^{e_{n}} G^{\prime}\right)=S_{\alpha}^{e_{1}+e_{2}+\ldots+e_{n}} G^{\prime},
$$

then $l_{\alpha}=S_{k_{1}}^{e_{1}} S_{k_{2}}^{e_{2}} \cdots S_{k_{n}}^{e_{n}} S_{\alpha}^{-p} \in G^{\prime}$. 
Since $S_{k_{1}}^{e_{1}} S_{k_{2}}^{e_{2}} \cdots S_{k_{n}}^{e_{n}}$ and $S_{k_{t}}^{e_{t}} S_{t+1}^{e_{t+1}} \ldots S_{k_{n}}^{e_{n}} S_{k_{1}}^{e_{1}} \ldots S_{t-1}^{e_{t-1}}$ represent the same lateral left class in $G_{a b}$, we assume that $l_{\alpha}=S_{k_{1}}^{e_{1}} S_{k_{2}}^{e_{2}} \cdots S_{k_{n}}^{e_{n}} S_{\alpha}^{-p}=$ $S_{k_{t}}^{e_{t}} S_{k_{t+1}}^{e_{t+1}} \ldots S_{k_{n}}^{e_{n}} S_{k_{1}}^{e_{1}} \ldots S_{k_{t-1}}^{e_{t-1}} S_{\alpha}^{-p}, t=1,2, \ldots, n$.

Definition 6. Let $K$ be a combinatorial knot. For $\alpha=1,2, \ldots, n$, each arc $S_{\alpha}$ of $K$ is called a meridian of $K$ and $l_{\alpha}$ is called a longitude of $K$. A peripheral pair of a combinatorial knot $K$ is the pair $\left(m_{\alpha}, l_{\alpha}\right)$.

In the classical knot theory the peripheral pair has an important geometric role, but in combinatorial knot theory we do not have any geometric interpretation yet.

Example 4. Let $K=((-1,2,-3,1,-2,3),(-1,-1,-1))$ a longitude for $K$ is $l=S_{1}^{-1} S_{2}^{-1} S_{3}^{-1} m^{3}$. Figure 7 shows how we can get such longitude in the classical case.
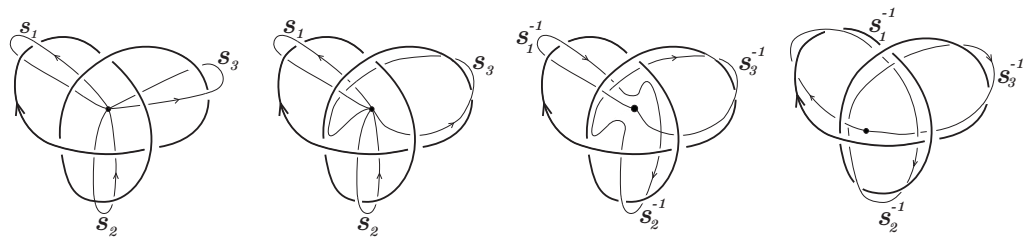

FiguRE 7. A longitude in a classical knot diagram.

We say that two peripheral pairs $\left(m_{i}, l_{i}\right)$ and $\left(m_{j}, l_{j}\right)$ are conjugate in $G(K)$ if there exists $w \in G(K)$ such that $m_{i}=w^{-1} m_{j} w$ and $l_{i}=w^{-1} l_{j} w$.

A peripheral structure of a combinatorial knot $K$ is the conjugated class in $G(K)$ of a peripheral pair of $K$.

Proposition 1. The peripheral structure of a combinatorial knot is unique up to conjugation.

Proof. Let $\left(S_{i}, l_{i}\right)$ and $\left(S_{j}, l_{j}\right)$ be two peripheral pairs of a combinatorial knot $K$. We may assume that $j>i$. From the relators $r_{i+1}, \ldots, r_{j}$ we get that

$$
S_{j}=S_{t_{j}}^{-e_{j}} S_{t_{j-1}}^{-e_{j-1}} \ldots S_{t_{i+1}}^{-e_{i+1}} S_{i} S_{t_{i+1}}^{e_{i+1}} \ldots S_{t_{j-1}}^{e_{j-1}} S_{t_{j}}^{e_{j}},
$$

where $v \in S_{t_{v}}, v=i+1, \ldots, j$, thus, if $w_{1}=w_{1}(i, j)=S_{t_{i+1}}^{e_{i+1}} \ldots S_{t_{j-1}}^{e_{j-1}} S_{t_{j}}^{e_{j}}$, then $S_{j}=w_{1}^{-1} S_{i} w_{1}$.

$$
l_{j}=S_{t_{j+1}}^{e_{j+1}} \ldots S_{t_{n}}^{e_{n}} S_{t_{1}}^{e_{1}} \ldots S_{t_{i}}^{e_{i}} S_{t_{i+1}}^{e_{i+1}} \ldots S_{t_{j}}^{e_{j}} S_{j}^{-p}=w_{2} w_{1} S_{j}^{-p},
$$


where $w_{2}=S_{t_{j+1}}^{e_{j+1}} \ldots S_{t_{n}}^{e_{n}} S_{t_{1}}^{e_{1}} \ldots S_{t_{i}}^{e_{i}}$. Since $S_{j}^{-p}=w_{1}^{-1} S_{i}^{-p} w_{1}$, then $l_{j}=$ $w_{2} S_{i}^{-p} w_{1}=w_{1}^{-1} w_{1} w_{2} S_{i}^{-p} w_{1}$. Now,

$$
l_{i}=S_{t_{i+1}}^{e_{i+1}} \ldots S_{t_{j-1}}^{e_{j-1}} S_{t_{j}}^{e_{j}} S_{t_{j+1}}^{e_{j+1}} \ldots S_{t_{n}}^{e_{n}} S_{t_{1}}^{e_{1}} \ldots S_{t_{i}}^{e_{i}} S_{i}^{-p}=w_{1} w_{2} S_{i}^{-p},
$$

so $l_{j}=w_{1}^{-1} l_{i} w_{1}$, therefore $\left(S_{i}, l_{i}\right)$ and $\left(S_{j}, l_{j}\right)$ are conjugates in $G(K)$.

Let $K$ be a combinatorial knot, $G(K)$ its group and $(m, l)=\left(S_{i}, l_{i}\right)$ the peripheral structure of $K$. The triplet $(G(K), m, l)$ is called peripheral system of $K$. We say that two peripheral systems $\left(G\left(K_{1}\right), m, l\right)$ and $\left(G\left(K_{2}\right), m^{\prime}, l^{\prime}\right)$ are isomorphic if and only if there exists an isomorphism $\varphi: G\left(K_{1}\right) \rightarrow G\left(K_{2}\right)$ such that $\varphi(m)=m^{\prime}$ and $\varphi(l)=l^{\prime}$. We have that the peripheral system is an invariant of combinatorial knots.

Waldhausen's theorem [24] implies that the equivalence class of classical knots is determined by the fundamental group and the peripheral system, see [9] for the proof.

Theorem 4. (Waldhausen) Two classical combinatorial knots $K_{1}$ and $K_{2}$, with peripheral structures $\left(G\left(K_{i}\right), l_{i}, m_{i}\right), i=1,2$, are equivalent if and only if there exists an isomorphism $\varphi: G\left(K_{1}\right) \rightarrow G\left(K_{2}\right)$ such that $\varphi\left(l_{1}\right)=l_{2}$ and $\varphi\left(m_{1}\right)=m_{2}$.

Corollary 1. Let $K$ be a classical combinatorial knot with trivial longitude, then $K$ is the trivial knot.

Example 5. For the combinatorial knot $K=((-1,2,3,-4,-3,4,1,-2)$, $(1,1,-1,-1))$ we have, $G(K) \supsetneqq \mathbb{Z}$. The longitude $l_{1}$ satisfies

$$
l_{1}=S_{3} S_{1} S_{1}^{-1} S_{3}^{-1} a_{2}^{0}=e .
$$

By Waldhausen's theorem $K$ is not classical.

4.3. Wirtinger Presentation. Let $G$ be a group. A Wirtinger presentation of $G$ is a presentation of the form

$$
G=\left\langle x_{1}, x_{2}, \cdots, x_{p}: r_{1}, r_{2}, \cdots, r_{q}\right\rangle,
$$

where $r_{k}=x_{j}^{w_{k}} x_{i}^{-1}, 1 \leq i, j \leq p$ and $w_{1}, \cdots, w_{q}$ are words in the free group $F\left(x_{1}, \ldots, x_{p}\right)$, not necessarily different in $G$.

A Wirtinger presentation is called cyclic if it is of the form

$$
\left\langle x_{1}, x_{2}, \cdots, x_{n}: r_{1}, r_{2}, \cdots, r_{m}\right\rangle,
$$

where $r_{i}=x_{i}^{w_{i}} x_{i+1}^{-1}, i=1,2, \ldots, m, w_{1}, \cdots, w_{m} \in F\left(x_{1}, \ldots, x_{n}\right)$, not necessarily different in $G$.

A Wirtinger presentation is called realizable if $w_{i}=x_{k_{i}}^{\varepsilon_{i}}$, for $i \in\{1,2, \ldots, n\}$ and $\varepsilon_{i} \in\{1,-1\}$. 
Note that the group of a combinatorial knot is given by a realizable Wirtinger presentation, see Definition 3.

Definition 7. The deficiency of a finite group presentation is the number of generators minus the number of relators. The deficiency of a group $G$, $\operatorname{def}(G)$, is defined as the maximum deficiency of all finite group presentation for $G$.

If $K$ is a combinatorial knot then $G(K)$ has a realizable Wirtinger presentation of deficiency 0 or 1.

The following result is central in the theory of virtual knots and its proof shows the advantage of working with combinatorial knots.

Theorem 5. Let $G$ be a group with realizable Wirtinger presentation of deficiency 0 , then there exist a combinatorial knot $K$ such that $G(K) \cong G$.

Proof. Let $G$ a group such that $G=\left\langle x_{1}, x_{2}, \cdots, x_{n}: r_{1}, r_{2}, \cdots, r_{n}\right\rangle$, with $r_{i}=x_{k_{i}}^{-e_{i}} x_{i} x_{k_{i}}^{e_{i}} x_{i+1}^{-1}, i=1,2,3, \ldots, n, x_{n+1}=x_{1}$ and $e_{i} \in\{1,-1\}$. Let $K$ the combinatorial knot given by $K=\left(\left(A_{1},-1, A_{2},-1, A_{3},-3, \ldots, A_{i},-i, A_{i+1}\right.\right.$, $\left.\left.\ldots, A_{n},-n\right)\left(e_{1}, e_{2}, \ldots, e_{n}\right)\right)$, where $\left\{A_{1}\right\}, \ldots,\left\{A_{n}\right\}$ are subset of $\{1,2, \ldots, n\}$ defined by $\left\{A_{i}\right\}=\left\{j \in\{1,2, \ldots, n\}: x_{k_{j}}^{e_{j}}=x_{i}^{ \pm 1}\right\}$. We have that $\cup_{i=1}^{n}\left\{A_{i}\right\}=$ $\{1,2, \ldots, n\}$. Is possible that some of $A_{i}$ are empty.

If $S_{1}=\left(-n, A_{1},-1\right), S_{2}=\left(-1, A_{2},-2\right), \ldots, S_{n}=\left(-(n-1), A_{n},-n\right)$, then $i \in S_{k_{i}}$ and

$$
G(K)=\left\langle S_{1}, S_{2}, \cdots, S_{n}: \mathbf{r}_{1}, \mathbf{r}_{2}, \cdots, \mathbf{r}_{n}\right\rangle,
$$

where $\mathbf{r}_{i}=S_{k_{i}}^{-e_{i}} S_{i} S_{k_{i}}^{e_{i}} S_{i+1}^{-1}, i=1,2, \ldots, n, S_{n+1}=S_{1}$. So $G(K) \cong G$.

Example 6. Let $G=\left\langle x_{1}, x_{2}, x_{3}: x_{1}^{x_{3}} x_{2}^{-1}, x_{2}^{x_{1}^{-1}} x_{3}^{-1}, x_{3}^{x_{2}^{-1}} x_{1}^{-1}\right\rangle$, then: $r_{1}=$ $x_{1}^{x_{3}} x_{2}^{-1}, r_{2}=x_{2}^{x_{1}^{-1}} x_{3}^{-1}$ and $r_{3}=x_{3}^{x_{2}^{-1}} x_{1}^{-1}$, so

$$
A_{1}=(2), A_{2}=(3) \text { and } A_{3}=(1) \text {. }
$$

therefore, if

$$
K=((-1,2,-2,3,-3,1),(-1,1,-1)),
$$

then $G(K)=G$. Moreover, a peripheral structure of $K$ is

$$
\left(x_{1}, x_{2}^{-1} x_{3} x_{1}^{-1} x_{1}\right)=\left(x_{1}, x_{2}^{-1} x_{3}\right) .
$$

Theorem 6. Let $G$ be a group with cyclic Wirtinger presentation of deficiency 0 or 1 , then there exists a combinatorial knot $K$ such that $G(K) \cong G$. 
Proof. Suppose that $G$ has a presentation of the form $\left\langle x_{1}, x_{2}, \cdots, x_{n}: r_{1}\right.$, $\left.r_{2}, \cdots, r_{n-1}\right\rangle$, where $r_{i}=w_{i}^{-1} x_{i} w_{i} x_{i+1}^{-1}, \quad i=1,2, \ldots, n-1$. Let $w_{n}=$ $\left(w_{1} w_{2} \ldots w_{n-1}\right)^{-1}$, then $G$ is isomorphic to $\left\langle x_{1}, x_{2}, \cdots, x_{n}: r_{1}, r_{2}, \cdots, r_{n-1}\right.$, $\left.\mathbf{r}_{n}\right\rangle$, with $\mathbf{r}_{n}=w_{n}^{-1} x_{n} w_{n} x_{1}^{-1}$. So, without loss of generality we may assume that $G$ has deficiency 0.

Suppose that $w_{i}=x_{i_{1}}^{\varepsilon_{1}} x_{i_{2}}^{\varepsilon_{1}} \ldots x_{i_{k}}^{\varepsilon_{k}}$, where $k=k(i)$, and $\varepsilon_{1}, \ldots, \varepsilon_{k} \in\{1,-1\}$, then

$$
x_{i+1}=x_{i_{k}}^{-\varepsilon_{k}} \ldots x_{i_{2}}^{-\varepsilon_{1}} x_{i_{1}}^{-\varepsilon_{1}} x_{i} x_{i_{1}}^{\varepsilon_{1}} x_{i_{2}}^{\varepsilon_{1}} \ldots x_{i_{k}}^{\varepsilon_{k}} .
$$

Let $y_{(i, 0)}=x_{i}, y_{(i, 1)}=x_{i_{1}}^{-\varepsilon_{1}} y_{(i, 0)} x_{i_{1}}^{\varepsilon_{1}}, \cdots, y_{(i, k-1)}=x_{i_{k-1}}^{-\varepsilon_{k-1}} y_{(i, k-2)} x_{i_{k-1}}^{\varepsilon_{k-1}}$. By Tietze transformation, $G$ is isomorphic to the presentation

$$
\left\langle x_{1}, x_{2}, x_{n}, y_{(1,0)}, \cdots, y_{(n, k(n))}: r_{1}, r_{2}, \cdots, r_{n}, R\right\rangle,
$$

where $R=\left\{y_{(i, 0)}=x_{i}, y_{(i, r)}^{-1} x_{i_{r}}^{-\varepsilon_{r}} y_{(i, r-1)} x_{i_{r}}^{\varepsilon_{r}}\right\}_{r=1, \ldots, k(i)-1}^{i=1, \ldots, n}$. thus,

$$
G \cong\left\langle y_{(1,0)}, \cdots, y_{(i, 0)}, \cdots, y_{(i, k(i)-1)}, \cdots, y_{(n, k(n))}: \mathbf{T}\right\rangle,
$$

with,

$$
\mathbf{T}=\left\{y_{(i, r)}^{-1} y_{\left(i_{r}, 0\right)}^{-\varepsilon_{r}} y_{(i, r-1)} y_{\left(i_{r}, 0\right)}^{\varepsilon_{r}}\right\}_{i=1,2, \ldots, n}^{r=1,2, \ldots, k(i)-1} .
$$

which is a realizable Wirtinger presentation, by Theorem 5 , there exists a combinatorial knot $K$ such that $G(K) \cong G$. From Theorem 5 , if $G$ is a group with a realizable Wirtinger presentation of deficiency 0 , then $G$ is the group of a combinatorial knot.

Example 7. Let $G=\left\langle x_{1}, x_{2}, x_{3}: x_{1}^{-1} x_{3}^{x_{2}^{2}}, x_{2}^{-1} x_{1}^{x_{3}^{2}}, x_{3}^{-1} x_{2}^{x_{1}^{2}}\right\rangle$.

Let $y_{1}=x_{3}, y_{2}=x_{2}^{-1} y_{1} x_{2}, y_{3}=x_{1}, y_{4}=x_{3}^{-1} y_{3} x_{3}, y_{5}=x_{2}, y_{6}=$ $x_{1}^{-1} y_{5} x_{1}$. Then $G$ has the following presentation,

$\left\langle y_{1}, y_{2}, y_{3}, y_{4}, y_{5}, y_{6}: y_{3}^{-1} y_{2}^{y_{5}}, y_{5}^{-1} y_{4}^{y_{1}}, y_{1}^{-1} y_{6}^{y_{3}}, y_{2}^{-1} y_{1}^{y_{5}}, y_{4}^{-1} y_{3}^{y_{1}}, y_{6}^{-1} y_{5}^{y_{3}}\right\rangle \cong$

$\cong\left\langle y_{1}, y_{2}, y_{3}, y_{4}, y_{5}, y_{6}: y_{1}^{-1} y_{6}^{y_{3}}, y_{2}^{-1} y_{1}^{y_{5}}, y_{3}^{-1} y_{2}^{y_{5}}, y_{4}^{-1} y_{3}^{y_{1}}, y_{5}^{-1} y_{4}^{y_{1}}, y_{6}^{-1} y_{5}^{y_{3}}\right\rangle$.

So, if $K=((-1,4,5-2,-3,1,6-4,-5,2,3-6),(1,1,1,1,1,1))$ then $G(K) \cong G$.

Theorem 7. If $G$ is a group with Wirtinger presentation of deficiency 0 or 1 such that $G_{a b} \cong \mathbb{Z}$, then $G$ is a combinatorial knot group.

Proof. Let $G=\left\langle x_{1}, x_{2}, \cdots, x_{p}: r_{1}, r_{2}, \cdots, r_{q}\right\rangle$ a Wirtinger presentation, where $q=p$ or $q=p-1$. By doubling any relators, we may assume that $q=p$.

Let $I(G)$ the graph such that its vertex are labeled with $x_{1}, x_{2}, \ldots, x_{p}$. $x_{i} x_{j}$ is a edge of $I(G)$ if and only if there exist a relator in $\left\{r_{1}, r_{2}, \ldots, r_{p}\right\}$ of 
the form $x_{i}^{w} x_{j}^{-1}$. Since $G_{a b} \cong \mathbb{Z}$, then all $x_{i}$ are conjugated in $G$, so $I(G)$ is a connected graph. Consider the operation on the graph shown in Figure 8 ,

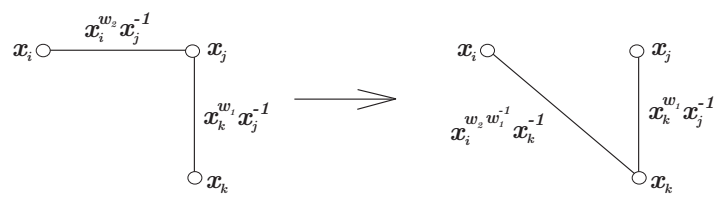

FiguRE 8. Operation on the graph $I(G)$

$I(G)$ can be transformed, using a finite number of the movements showed in Figure 8, in a cycle $C_{p}$ such that $V\left(C_{p}\right)=V(I(G))$ and $E\left(C_{p}\right)=$ $E(I(G))$, see [17]. Suppose that $C_{p}=x_{i_{1}} x_{i_{2}} \ldots x_{i_{p}} x_{i_{1}}$, if we denote $y_{t}=x_{i_{t}}$, $t=1,2, \ldots, p$, then $C_{p}=y_{1} y_{2} \ldots y_{p} y_{1}$ and the corresponding operations on the relators of $G$ give a cyclic Wirtinger presentation. By Theorem $6 G$ is a combinatorial knot group.

Example 8. The Baumslag-Solitar group $G=\left\langle x, y: x^{-1} y^{2} x=y^{3}\right\rangle$ is the group of a combinatorial knot. Since

$$
G \cong\left\langle x, y, y_{1}, y_{2}: y=x^{-1} y^{2} x y^{-2}, y_{1}=x, y_{2}=y^{2} x y^{-2}\right\rangle,
$$

then $G \cong\left\langle y_{1}, y_{2}: y_{2}=y_{1}^{\left(y_{1}^{-1} y_{2}\right)^{-2}}, y_{1}=y_{2}^{\left(y_{1}^{-1} y_{2}\right)^{2}}\right\rangle$.

If $x_{1}=y_{1}, x_{2}=y_{2} x_{1} y_{2}^{-1}, x_{3}=y_{1} x_{2} y_{1}^{-1}, x_{4}=y_{2} x_{3} y_{2}^{-1}, x_{5}=y_{2}, x_{6}=$ $y_{1}^{-1} x_{5} y_{1}, x_{7}=y_{2}^{-1} x_{6} y_{2}$ and $x_{8}=y_{1}^{-1} x_{7} y_{1}$, then

$G=\left\langle x_{1}, x_{2}, x_{3}, x_{4}, x_{5}, x_{6}, x_{7}, x_{8}: x_{8}^{x_{5}} x_{1}^{-1}, x_{1}^{x_{5}^{-1}} x_{2}^{-1}, x_{2}^{x_{1}^{-1}} x_{3}^{-1}, x_{3}^{x_{5}^{-1}} x_{4}^{-1}\right.$, $\left.x_{4}^{x_{1}^{-1}} x_{5}^{-1}, x_{5}^{x_{1}} x_{6}^{-1}, x_{6}^{x_{5}} x_{7}^{-1}, x_{6}^{x_{5}} x_{7}^{-1}\right\rangle$.

So $G$ is the group of the combinatorial knot

$K=((-1,3,5,6,8,-2,-3,-4,-5,1,2,4,7,-6,-7,-8),(1,-1,-1,-1$, $-1,1,1,1))$.

The corresponding virtual knot is given in Figure 9 . 


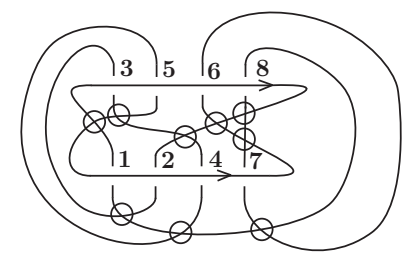

FiguRE 9. A virtual knot for the Baumslag-Solitar group

\section{References}

[1] Burde, G. and Zieschang, H.,. Knots. Walter de Gruyter, New York, NY (1985)..

[2] Carter, J., Classifying Immersed Curves, Proc. Amer. Math. Soc. 111, No. 1 (1991), 281-287.

[3] Cairns, G. and Elton, D., The Planarity Problem for Signed Gauss Words, Journal of Knot Theory and its Ramifications, Vol. 2, No. 4 (1993) 359-367.

[4] Carter, S. Kamada, S. and Saito, M., Stable Equivalence of Knots on surfaces and Virtual Knots Cobordism, Journal of Knot Theory and its Ramifications, Vol. 11, No. 6 (2002), 311-320.

[5] Cotta-Ramusino, P. and Rinaldi, M., On the Algebraic Structure of Link-Diagrams on a 2-Dimensional Surface, Communications in Mahematical Physics, $131,137-173(11)$.

[6] Crowell, R and Fox, R., Introduction to Knot Theory, Blaisdell Publishing Company, New York, 1963.

[7] Dye, H. Kauffman, L., Minimal Surface representations of virtual Knots and Links, Algebraic and Geometric Topology, Vol. 5, (2005), 509-535.

[8] Goussarov, M. Polyak, M. and Viro, O., Finite Type Invariants of Classical and Virtual Knots, Preprint: math. GT/1981/9810073.

[9] Hempis, J., 3-manifolds, Ann. of Math. Studios, 86, Princeton Univ. Press.

[10] Henrich, A., Ph.D. thesis, expected in Spring (2008).

[11] Hilden, M. H., Montesinos, J. M., Tejada, D. M. and Toro, M. M., Mariposas and 3-variedades. Rev. Acad. Colomb. Cienc. 28(106), 71-78, 2004.

[12] Jaeger, F., Composition Products and Models for the HOMFLY polynomials, Enseign. Math. 35 (1989), 323-361.

[13] Jaeger, F., Strongly Regular Graphs and Spin models for the Kauffman Polynomials, Geom. Dedicata 44 (1992), 23-52.

[14] Jaeger, H. Kauffman, L. and Saleur, H., The Conway polynomials in $R^{3}$ and in Thickened surfaces: A New Determinant Formulation, Journal of Combinatorial theory, Series B 61, 237-259 (1994).

[15] Kamada, N. and Kamada, S., Abstract Links Diagrams and Virtual Knots, Journal of Knot Theory and its Ramifications, Vol. 9, No. 1 (2000), 93-106.

[16] Kauffman, L., Virtual Knot theory, Europ. J. Combinatorics vol. 20 (1999) 663691.

[17] Kim, S., Virtual Knot Groups and their Peripheral Structure, Journal of Knot Theory and its Ramifications, Vol. 9, No. 6 (2000), 797-812.

[18] Kupenberg, G., What is a Virtual Knots?, Algebraic and Geometric Topology, Vol. 3, (2003), 587-591.

[19] Read, R. and Rosenstiehl, P., On the Gauss Crossing Problems, Coll.Math.Soc. Janos Bolyai 18 (1976), 843-876. 
[20] Silver, D. and Williams, S., Virtual Knots Groups, Preprint (1999).

[21] Toro, M. M., Nudos Combinatorios y Mariposas. Rev. Acad. Colomb. Cienc. 28(106), 79-86, 2004.

[22] Toro, M.M. and Rodríguez, J. G., Nudos combinatorios: Una nueva visión de los nudos virtuales, preprint, 2008.

[23] Turaev, V., Cobordism of knots on surfaces, preprint arXiv:math/0703055 available at http://www.arXiv.org, 28 pages (2007)

[24] Waldhausen, F., On Irreducible 3-manifold which are sufficiently large, Ann. of Math., 87: 56-88 (1968) 